\title{
The outcome measures for loss of functionality in the activities of daily living of adults after stroke: $a$ systematic review
}

\author{
Galeoto Giovanni, Iori Francesca, De Santis Rita, Santilli Valter, Mollica \\ Roberta, Maria Auxiliadora Marquez, Julita Sansoni \& Anna Berardi
}

To cite this article: Galeoto Giovanni, lori Francesca, De Santis Rita, Santilli Valter, Mollica Roberta, Maria Auxiliadora Marquez, Julita Sansoni \& Anna Berardi (2019): The outcome measures for loss of functionality in the activities of daily living of adults after stroke: a systematic review, Topics in Stroke Rehabilitation

To link to this article: https://doi.org/10.1080/10749357.2019.1574060

\section{Published online: 18 Feb 2019.}

Submit your article to this journal ${ }^{3}$

View Crossmark data ¿ 


\title{
The outcome measures for loss of functionality in the activities of daily living of adults after stroke: a systematic review
}

\author{
Galeoto Giovanni (10), Iori Francescab ${ }^{b}$, De Santis Ritac ${ }^{c}$, Santilli Valter ${ }^{c}$, Mollica Robertac, Maria Auxiliadora Marquez ${ }^{\mathrm{d}}$, \\ Julita Sansoni ${ }^{\mathrm{d}}$ and Anna Berardi ${ }^{\mathrm{b}}$

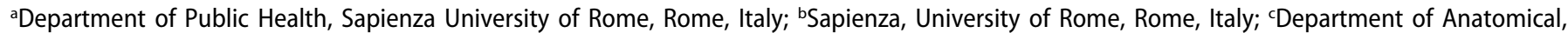

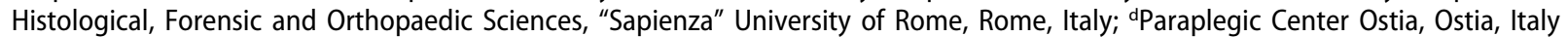

\section{ABSTRACT}

Objective: To provide clinicians and researchers information regarding (1) the existing outcome measures to assess the loss of functionality in the activities of daily living (ADLs) of patients with stroke and (2) the presence of these assessment tools in the Italian context.

Study Design and Setting: For this Systematic Review Medline, CINAHL, and PsycINFO were searched for articles published up to 4 July 2017. Two authors independently identified eligible studies on the basis of predefined inclusion criteria and extracted data. Study quality and risk of bias were assessed using the Quality Assessment Tool for Observational Cohort and Cross-Sectional Studies.

Results: Of 370 publications identified and screened, 46 studies fell within the inclusion criteria and were critically reviewed. The most commonly used tools were: the Frenchay Activities Index and the Functional Independence Measure.

Conclusion: This review has emphasized the need for agreement among researchers as to which tool must be studied in depth or adapted to other national contexts in order to develop universal norms and standards.
ARTICLE HISTORY

Received 26 August 2018

Accepted 19 January 2019

\section{KEYWORDS}

$\mathrm{ADL}$; questionnaire;

reliability; review;

psychometric properties; stroke rehabilitation; tools; validation

\section{Introduction}

During the acute phase after stroke, treatment focuses on specialized medical intervention and continuous rehabilitation for a long period of time. ${ }^{1}$ Patients may experience reduced motor and cognitive function, difficulties in performing daily activities, and a reduced health-related quality of life. ${ }^{2,3}$ Health professionals and rehabilitation professionals often use a person's ability or inability to perform activities of daily living as a measurement of their functional status, this focus on rehabilitation after a stroke, especially in occupational therapy, aims to identify the best treatment methods and routines to help patients return to their previous lives and daily activities. The use of the term "activities of daily living" (ADLs) is familiar to many, however, universal agreement of the concept and definition of ADL has been problematic, with subdivision of ADL into basic or personal ADLS (BADL,PADL) and instrumental or extended ADLs (IADL, EADL). Activities of Daily Living (ADLs) are defined by the Medical Dictionary as "the things we normally do... such as feeding ourselves, bathing, dressing, grooming, work, homemaking, and leisure". ${ }^{4}$ Consistent with the Medical Dictionary's definition we have considered for ADLs including basic, personal, instrumental, and extended activities of daily living.

Thus, to identify the best treatment methods and routines to help patients return to their previous lives and daily activities the first step is to identify the correct assessment tool to robustly assess the efficacy of interventions both at the level of the individual stroke survivor and in the context of clinical trials.
The classic clinical trial is designed to test the efficacy of a particular intervention as compared to another intervention or a control group. Facilitating comparison between groups requires a standard measure of outcome that is relevant and suited to the clinical question, valid for the population studied, and meaningful to the research team. ${ }^{5}$ Therefore it was decided to do this systematic review because clinicians and researchers need to know the most reliable, valid, and universally accepted measures currently available for addressing this clinical construct in stroke survivors and to allow comparisons between different rehabilitation programs.

Systematic reviews and meta-analyses have become increasingly important in health care. Clinicians read them to keep up to date with their field $d^{6,7}$ and they are often used as a starting point for developing clinical practice guidelines. ${ }^{8-14}$ Of course, within a single review, it would be impossible to analyze all the scales that may be needed on a stroke survivor's journey, a number of reference works exist in literature for stroke-specific and generic scales, ${ }^{15-17}$ but none of these analyzes the loss of functionality on ADLs. In the literature, a wide range of tools have been used to evaluate the loss of ADL functionality on various ADL domains in patients with stroke, and no broadly accepted consensus as to which are preferred has been reached.

This study aimed to provide clinicians and researchers information regarding the existing outcome measures to assess the loss of functionality in the activities of daily living (ADLs) of patients with stroke and the presence of these

CONTACT Galeoto Giovanni giovanni.galeoto@uniroma1.it Department of Public Health, Sapienza University of Rome, Piazzale Aldo Moro 5, Rome 00185, Italy Color versions of one or more of the figures in the article can be found online at www.tandfonline.com/ytsr.

(๑) 2019 Taylor \& Francis Group, LLC 
assessment tools in the Italian context (culture, rehabilitation practices and language) based on reviewing, analyzing, comparing, and critically appraising the available outcome measures and their distribution in the international literature.

\section{Methods}

\section{Protocol and registration}

After registering the protocol on the Prospero website, the international prospective register of systematic reviews, available at http://www.crd.york.ac.uk/PROSPERO/display_ record.php?ID=CRD42017076815, this review was carried out in accordance with the 27-item PRISMA Statement for Reporting Systematic Reviews ${ }^{9,10}$ on the basis of MECIR. ${ }^{6-8}$

\section{Eligibility criteria for considering studies for this review: types of studies and types of participants}

A systematic review of the English-language literature was undertaken by looking for studies that evaluated the psychometric properties of outcome measures that explored the loss of functionality in the ADLs of adults with a history of stroke. Consistent with the Medical Dictionary's definition we have considered for ADLs "the things we normally do... such as feeding ourselves, bathing, dressing, grooming, work, homemaking, and leisure", including basic, personal, instrumental, and extended activities of daily living. All studies that evaluated the psychometric properties of a clinician's report, a patient's self-report, and/or physical performance outcome measures that measured functionality in the ADLs of patients in at least one domain of the tool were included. Studies were limited to people with stroke, regardless of clinical course or length of time since diagnosis. Studies with mixed diagnosis samples were included if a subgroup of participants could be identified and for which separate data were available. Studies were included if participants were 18 years or over or if separate data were available for those over 18 years old. No restrictions were applied to the publication period or to the country in which the study was conducted but the searches were limited to studies published in English.

\section{Inclusion criteria}

(1) Validation studies and cross-cultural adaptation studies;

(2) Studies about the loss of functionality in active ADLs and IADLs performance;

(3) Studies about tests, questionnaires, and self-reported and performance-based outcome measures;

(4) Studies with a population of patients with stroke;

(5) Population $\geq 18$ years old; and

(6) Studies published in English.

\section{Exclusion criteria}

(1) Trials or studies that evaluated the effectiveness of interventions where a questionnaire is used as an endpoint (without studying the measurement properties);

(2) Studies including measures that measure cognitive, motor and other constructs;
(3) Studies with mixed diagnosis samples if a subgroup of stroke participants could not be identified with separate data; or

(4) Studies with mixed age samples if a subgroup of adult participants could not be identified with separate data.

\section{Search methods for identification of the studies}

Studies were identified for inclusion through individualized systematic searches of three electronic databases. All potential studies were identified by two reviewers.

\section{Electronic searches}

The review's primary reviewer developed the search strategy, following consultation with an expert and using guidance from relevant past reviews. The initial search strategy was constructed for MEDLINE (via PubMed) on 4 July 2017. A combination of terms and keywords was used: (("stroke") AND "activities of daily living") AND "validation") AND ((("scale") OR "questionnaire") OR "test") and adapted to other databases. The following electronic databases were searched:

(1) Medline (via PubMed);

(2) CINAHL (via EBSCO); and

(3) PsycINFO (via EBSCO).

\section{Study selection}

Titles, keywords, and abstracts identified through the databases were screened independently by two occupational therapists. After the first screening, the primary reviewr selected the relevant studies and assessed them against the inclusion criteria. Then, a second reviewer cross-checked the studies. After the second screening, studies were systematically excluded that did not fit the inclusion criteria and others were identified that appeared pertinent. A final list of studies that were eligible for inclusion was compiled, and any disagreements were resolved by the third reviewr or by consensus. The studies that met the criteria were then subject to a full text review to select studies to decide whether or not to include them in the review.

\section{Data collection, data items, and assessment of risk of bias}

The approach to data extraction was chosen on the basis of the Cochrane Methods. ${ }^{18}$ Two reviewers independently extracted patient demographics and descriptive information and each study was keyworded for generic issues such as language, country, focus, population, and so on. ${ }^{19}$ These characteristics were judged on the basis of the information provided in the reports on the studies. For the data collection, we followed the recommendations from the COSMIN initiative, ${ }^{20-23}$ which takes into account the special aspects of validating patient-reported outcome measures (PROMs). Outcome measures reported within each publication were recorded and categorized for comparison. We decided to report the following psychometric characteristics: Cronbach's alpha for internal consistency, intraclass correlation coefficient 
for stability, and Pearson's correlation for validity. The studies' content and methodology were analyzed qualitatively. Aspects of validity were defined and a checklist was used to determine which aspects had been evaluated. Study quality and risk of bias were assessed using the Quality Assessment Tool for Observational Cohort and Cross-Sectional Studies (QAT). ${ }^{24}$ The QAT scale is composed of 14 items; 2 of these (items 6 and 7) are used exclusively for observational cohort studies, which were not included in this review. Therefore, each study in the review was evaluated with the remaining 12 items.

\section{Results}

Study selection: description of the studies and results of the search

The search strategy identified 370 results. After removing duplicates, 329 articles were screened by reading titles and abstracts, resulting in 106 papers that were screened further by reading the full text. Figure 1 provides a flowchart demonstrating the search and screening process.

\section{Excluded studies}

In total, 283 studies were excluded on the basis of the exclusion criteria. During the title and abstract screening, 223 studies were excluded because they had not studied specific outcome measures that assessed the loss of functionality in the ADLs of patients with stroke (i.e quality of life outcome measures). Of the studies that received a full text review, 67 articles were excluded; of these, 34 were studies that evaluated the effectiveness of interventions where a questionnaire was used as an endpoint, 25 were not active ADL's performance specific, 7 had mixed diagnosis samples without separate data for a stroke subgroup, and 1 was not published in English.

\section{Included studies}

Out of the $39^{25-63}$ articles that fell within the inclusion criteria and were critically reviewed, $2^{33,43}$ validated two measurement tools at the same time. A total of 41 tools were identified and 6 of these were validated by multiple studies. We found 33 different outcome measures that assessed the loss of functionality in the ADLs of patients with stroke. A summary of the studies' descriptive information is presented in Table 1.

\section{Study characteristics: types of design and types of participants}

All the studies were cross-sectional. ${ }^{25-63}$ The sample size in the studies varied from $17^{34}$ to $603 .^{30}$ The majority of the participants were in their mid to late $60 \mathrm{~s}$, with mean ages ranging from $53 \pm 13$ years ${ }^{56}$ to $77.8 \pm 7.5$ years. ${ }^{35}$ The most represented languages were Chinese $\mathrm{C}^{27,28,36,40-43,53,62}$ and

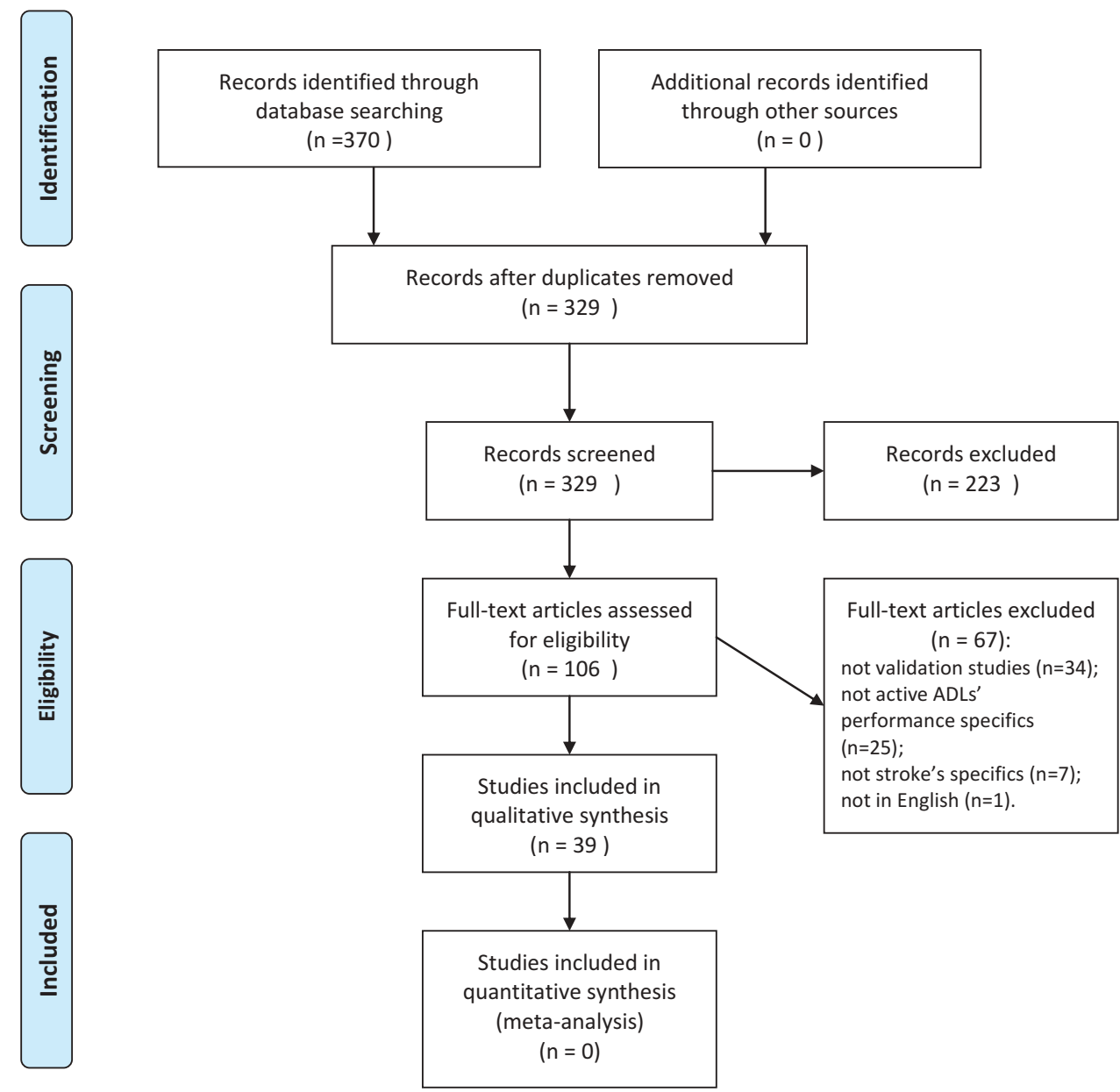

Figure 1. Flowchart of search and screening process. 
Table 1. Descriptive information of included studies.

\begin{tabular}{|c|c|c|c|c|c|c|}
\hline Scale & Authors & Language & Items & $\begin{array}{l}\text { Sample } \\
\text { size }\end{array}$ & $\begin{array}{l}\text { mean } \pm S D \\
\text { age }\end{array}$ & Administration \\
\hline Abilhand questionnaire & Wang et al. 2011 & Chinese & 23 & 51 & $55.26 \pm 10.31$ & Test \\
\hline Ability Scale & Lee et al. 2014 & Chinese & 8 & 306 & $61 \pm 13.8$ & Test \\
\hline \multirow[t]{2}{*}{ Activity Card Sort } & Chan et al. 2006 & Chinese & 65 & 60 & 74 & Self-reported \\
\hline & Katz et al. 2003; & Israeli & 88 & 56 & $57.7 \pm 11.6$ & Self-reported \\
\hline ACTIVLIM-Stroke & Batcho et al. 2012 & $\begin{array}{l}\text { French (Belgium } \\
\text { and Benin) }\end{array}$ & 20 & 204 & $57.1 \pm 13.4$ & Questionnaire \\
\hline $\begin{array}{l}\text { Canadian Occupational Performance Measure in stroke } \\
\text { patients }\end{array}$ & Cup et al. 2003 & Dutch & 5 & 26 & $68 \pm 15$ & Questionnaire \\
\hline $\begin{array}{l}\text { Combined Measure of Basic and Extended Daily Life } \\
\text { Functioning After Stroke }\end{array}$ & Chen et al. 2013 & Chinese & 39 & 188 & $55,96 \pm 11,7$ & Questionnaire \\
\hline Comprehensive Activities of Daily Living & Hsueh et al. 2012 & Chinese & 23 & 168 & $65.4 \pm 10.3$ & Questionnaire \\
\hline \multirow[t]{2}{*}{ Daily Living Self-Efficacy Scale } & Li et al. 2016 & Chinese & 12 & 172 & $63.39 \pm 11.06$ & Questionnaire \\
\hline & Maujean et al. 2013; & Australian English & 12 & 424 & $65.25 \pm 12.65$ & Questionnaire \\
\hline \multirow[t]{2}{*}{ Extended Activities of Daily Living } & Gompertz et al. 1994 & English & 22 & 159 & N.R.* & Self-reported \\
\hline & Lincoln et al. 1992; & English & 22 & 352 & 67 & Self-reported \\
\hline $\begin{array}{l}\text { Extended Barthel Index with Acute Ischemic Stroke } \\
\text { Patients }\end{array}$ & Jansa et al. 2004 & Slovenian & 16 & 33 & 62 & Test \\
\hline \multirow[t]{4}{*}{ Frenchay Activities Index } & Lin et al. 2012; & Chinese & 15 & 127 & $55.27 \pm 11.73$ & Questionnaire \\
\hline & Post et al. 2003; & Dutch & 15 & 35 & $71,1 \pm 14,8$ & Questionnaire \\
\hline & Piercy et al. 2000; & English & 15 & 45 & $55.6 \pm 10.9$ & Questionnaire \\
\hline & Monteiro et al. 2017 & Brazilian & 15 & 161 & $57.3 \pm 17.0$ & Questionnaire \\
\hline \multirow[t]{2}{*}{ Functional Independence Measure } & Naghdi et al. 2015 & Persian & 18 & 40 & $60 \pm 14.9$ & Test \\
\hline & Brosseau et al. 1996; & Canadian & 18 & 152 & $69 \pm 14$ & Test \\
\hline $\begin{array}{l}\text { Functional Indipendence Measure and Functional } \\
\text { Assessment Measure }\end{array}$ & Miki et al. 2015 & Japanese & 30 & 42 & $64.9 \pm 14.6$ & Questionnaire \\
\hline Human Activity Profile & $\begin{array}{l}\text { Teixeira-Salmela et al. } \\
2007\end{array}$ & Brazilian & 94 & 24 & $63.69 \pm 11.57$ & $\begin{array}{l}\text { Questionnaire/self- } \\
\text { reported/test }\end{array}$ \\
\hline Impact on Participation and Autonomy & Fallahpour et al. 2011 & Persian & 26 & 102 & $58,3 \pm 11,9$ & Self-reported \\
\hline Mayo-Portland Adaptability Inventory 4 & Hamed et al. 2012 & Arabic & 35 & 17 & $54.29 \pm 3.91$ & Questionnaire \\
\hline Modified Barthel Index & Leung et al. 2007 & Chinese & 10 & 116 & $76 \pm 7,6$ & Test \\
\hline Motor Activity Log & $\begin{array}{l}\text { Van der Lee et al. } \\
\text { 2004; }\end{array}$ & Dutch & 26 & 56 & 61 median & Questionnaire \\
\hline Motor Activity Log-28 & Uswatte et al. 2006 & American English & 30 & 222 & $62,2 \pm 13,0$ & Test/questionnaire \\
\hline Motor Functional Independence Measure & $\begin{array}{l}\text { Lundgren-Nilsson et al. } \\
\qquad 2006\end{array}$ & Scandinavian & 13 & 157 & N.R.* & Questionnaire \\
\hline $\begin{array}{l}\text { New Lucerne ICF Based Multidisciplinary Observation } \\
\text { Scale }\end{array}$ & Ottiger et al. 2015 & Swiss & 45 & 102 & $69,3 \pm 11,9$ & Questionnaire \\
\hline Northwick Park ADL Index & Spencer et al. 1986 & Australian English & 17 & N.R.* & N.R.* & Test \\
\hline Nottingham Leisure Questionnaire & Drummond et al. 2000 & English & 30 & 603 & N.R.* & Questionnaire \\
\hline Occupational Gaps Questionnaire & Fallahpour et al. 2011 & Persian & 31 & 102 & $58,4 \pm 11,9$ & Questionnaire \\
\hline Perceived Life Satisfaction & Fallahpour et al. 2011 & Persian & 11 & 102 & $58,4 \pm 11,9$ & Questionnaire \\
\hline \multirow[t]{2}{*}{ Reintegration to Normal Living Index } & Tooth et al. 2003; & Australian English & 11 & 57 & $70 \pm 12$ & Questionnaire \\
\hline & Pang et al. 2011 & Chinese & 11 & 75 & $64.4 \pm 12.3$ & Questionnaire \\
\hline Rivermead Activity of Daily Living Scale & Lincoln et al. 1990 & English & 31 & 105 & 65 & Questionnaire \\
\hline Self-perceived Difficulty Scale & Lee et al. 2014 & Chinese & 10 & 306 & $61 \pm 13.8$ & Questionnaire \\
\hline Short Version of the Motor FIM & Yamada et al. 2006 & Japanese & 7 & 356 & 70:07:00 & Questionnaire \\
\hline Stroke Activity Scale & Horgan et al. 2006 & Irish & 5 & 41 & $77.8 \pm 7.5$ & Test \\
\hline Sunnaas Index of Activities of Daily Living & Korpelainen et al. 1997 & Finnish & 12 & 55 & $59,07 \pm 11$ & Test \\
\hline Vision-Dependent Activities of Daily Living Instrument & Mennem et al. 2012 & American English & 38 & 30 & $63.8 \pm 8.1$ & Self-reported \\
\hline $\begin{array}{l}\text { World Health Organisation Disability Assessment } \\
\text { Schedule II }\end{array}$ & Schlote et al. 2009 & German & 36 & 77 & $53 \pm 13$ & Questionnaire \\
\hline
\end{tabular}

${ }^{*}$ N.R. Not Reported.

English, ${ }^{31,34,45,46,55}$ with 10 and 5 validated tools, respectively. The measurement properties for each version of each tool are summarized in Table 2 .

The most commonly used tools were: The Frenchay Activities Index (FAI), validated in Chinese, ${ }^{43}$ Brazilian, ${ }^{50}$ English, ${ }^{54}$ and Dutch $^{55}$; the Activity Card Sort (ACS), validated in Chinese ${ }^{27}$ and Israeli $^{38}$; the Daily Living Self-Efficacy Scale (DLSES), validated in Chinese $^{42}$ and Australian English ${ }^{47}$; the Reintegration to Normal Living Index (RNLI), validated in Chinese ${ }^{53}$ and Australian English $^{59}$; the Functional Independence Measure (FIM), validated in French-Canadian ${ }^{26}$ and Persian. ${ }^{51}$ Additionally, two further studies included the FIM instrument, one ${ }^{49}$ in Japanese validating its use in combination with the Functional Assessment Measure (FAM) scale, the other study ${ }^{46}$ validating only the motor subscale in the Scandinavian language. The Extended Activities of Daily
Living (EADL) is a tool whose measurement properties have been studied by two English authors in two different research studies. $^{33,45}$ The Lincoln et al. ${ }^{45}$ study only evaluated content validity through the reproducibility and scalability coefficients. Gompertz et al. ${ }^{33}$ investigated construct validity by comparing it with other comparable outcome measures, expressing that relationship through the Spearman Rho's value.

\section{Risk of bias within studies}

The risk of bias in the $39^{25-63}$ included studies was mixed. The quality scores from the responsiveness subset questions of the $\mathrm{QAT}^{24}$ checklist are reported in Table 3. In general, the studies were found to have fairly good quality. Items 1(Was the research question or objective in this paper clearly 
Table 2. Measurement properties of included studies.

\begin{tabular}{|c|c|c|c|c|}
\hline Authors & Scale & ICC & R-PEARSON & a-CRONBACH \\
\hline Wang et al. 2011 & Abilhand questionnaire & 0.87 & 0.66 & 0.88 \\
\hline Lee et al. 2014 & Ability Scale & N.R.* & N.R.* & N.R.* \\
\hline Chan et al. 2006 & Activity Card Sort & 0.98 & 0.86 & 0.89 \\
\hline Katz et al. 2003; & & N.R.* & N.R.* & 0.72 \\
\hline Batcho et al. 2012 & ACTIVLIM-Stroke & 0.92 & 0.83 & 0.95 \\
\hline Cup et al. 2003 & Canadian Occupational Performance Measure in stroke patients & N.R.* & N.R.* & N.R.* \\
\hline Chen et al. 2013 & Combined Measure of Basic and Extended Daily Life Functioning After Stroke & 0,91 & N.R.* & 0.94 \\
\hline Hsueh et al. 2012 & Comprehensive Activities of Daily Living & 0.96 & 0.75 & 0.94 \\
\hline Li et al. 2016 & Daily Living Self-Efficacy Scale & N.R.* & 0.87 & 0.96 \\
\hline Maujean et al. 2013; & & 0.98 & 0.59 & 0.95 \\
\hline Gompertz et al. 1994 & Extended Activities of Daily Living & N.R.* & N.R.* & N.R.* \\
\hline Lincoln et al. 1992; & & N.R.* & N.R.* & N.R.* \\
\hline Jansa et al. 2004 & Extended Barthel Index with Acute Ischemic Stroke Patients & N.R.* & N.R.* & 0.94 \\
\hline Lin et al. 2012; & Frenchay Activities Index & N.R.* & 0.99 & 0.88 \\
\hline Post et al. 2003; & & N.R.* & N.R.* & N.R.* \\
\hline Piercy et al. 2000; & & 0.90 & N.R.* & N.R.* \\
\hline Monteiro et al. 2017 & & 0.82 & N.R.* & 0.99 \\
\hline Naghdi et al. 2015 & Functional Independence Measure & 0.98 & 0.95 & 0.96 \\
\hline Brosseau et al. 1996; & & N.R.* & N.R.* & 0.96 \\
\hline Miki et al. 2015 & Functional Indipendence Measure and Functional Assessment Measure & N.R.* & 0.73 & 0.95 \\
\hline Teixeira-Salmela et al. 2007 & Human Activity Profile & 0.89 & 0.99 & N.R.* \\
\hline Fallahpour et al. 2011 & Impact on Participation and Autonomy & N.R.* & 0.75 & N.R.* \\
\hline Hamed et al. 2012 & Mayo-Portland Adaptability Inventory 4 & N.V & 0.73 & N.R.* \\
\hline Leung et al. 2007 & Modified Barthel Index & N.R.* & 0,91 & 0,93 \\
\hline Van der Lee et al. 2004; & Motor Activity Log & N.R.* & N.R.* & 0.88 \\
\hline Uswatte et al. 2006 & Motor Activity Log-28 & 0,82 & 0.72 & 0.94 \\
\hline Lundgren-Nilsson et al. 2006 & Motor Functional Independence Measure & N.R.* & N.R.* & N.R.* \\
\hline Ottiger et al. 2015 & New Lucerne ICF Based Multidisciplinary Observation Scale & 0.9 & 0.9 & 0.98 \\
\hline Spencer et al. 1986 & Northwick Park ADL Index & N.R.* & N.R.* & N.R.* \\
\hline Drummond et al. 2000 & Nottingham Leisure Questionnaire & N.R.* & N.R.* & N.R.* \\
\hline Fallahpour et al. 2011 & Occupational Gaps Questionnaire & N.R.* & 0.46 & N.R.* \\
\hline Fallahpour et al. 2011 & Perceived Life Satisfaction & N.R.* & 0.46 & N.R.* \\
\hline Tooth et al. 2003; & Reintegration to Normal Living Index & 0.36 & 0.65 & 0.80 \\
\hline Pang et al. 2011 & & 0.87 & 0.43 & 0.92 \\
\hline Lincoln et al. 1990 & Rivermead Activity of Daily Living Scale & N.R.* & N.R.* & N.R.* \\
\hline Lee et al. 2014 & Self-perceived Difficulty Scale & N.R.* & N.R.* & N.R.* \\
\hline Yamada et al. 2006 & Short Version of the Motor FIM & 0.99 & N.R.* & N.R.* \\
\hline Horgan et al. 2006 & Stroke Activity Scale & N.R.* & 0.91 & N.R.* \\
\hline Korpelainen et al. 1997 & Sunnaas Index of Activities of Daily Living & N.R.* & 0,88 & 0.95 \\
\hline Mennem et al. 2012 & Vision-Dependent Activities of Daily Living Instrument & N.R.* & 0.99 & 0,99 \\
\hline Schlote et al. 2009 & World Health Organisation Disability Assessment Schedule II & 0.67 & N.R.* & 0.97 \\
\hline
\end{tabular}

*N.R. Not Reported.

stated?), 9(Were the exposure measures (independent variables) clearly defined, valid, reliable, and implemented consistently across all study participants?), and 11(Were the outcome measures -dependent variables- clearly defined, valid, reliable, and implemented consistently across all study participants?) had the highest number of positive responses. The less represented items were 2 (Was the study population clearly specified and defined?), 5(Was a sample size justification, power description, or variance and effect estimates provided?), and 13 (Was loss to follow-up after baseline $20 \%$ or less?).

\section{Results of individual studies}

\section{Study population}

About half of the studies, 20, described the group of people from which the study participants were selected or recruited, using demographics, locations, and time periods. In the other studies, the authors did not report information about when and from where the population was recruited. In addition, only $10^{26,30-32,42,45,50,56,58,61}$ of the analyzed studies contained the percentage of eligible people who participated in the study. If fewer than $50 \%$ of the people who were eligible participated in the study, then there is a concern that the study population does not adequately represent the target population.

\section{Groups recruited from the same population; uniform eligibility criteria; sample size justification}

In 32 studies, the inclusion and exclusion criteria were developed prior to the recruitment of the study population and the same criteria were used for all of the subjects involved. Two studies $^{38,63}$ did not provide sufficient information to determine the inclusion and exclusion criteria development methods. In only 2 studies ${ }^{58,62}$ did the authors present their reasons for recruiting the number of people included and discuss the statistical power of the study. In the other studies, 37 , it was not being possible to determine if the study had enough participants.

\section{Different levels of the exposure of interest; exposure} measures and assessment; repeated exposure assessment; blinding of outcome assessors

In 8 studies ${ }^{26,27,33,36,38,58,62,63}$ we found a stratification for pathology levels. In almost all the studies, the authors used accurate and reliable measurement tools for the detection of 
Table 3. Quality scores of the included studies.

\begin{tabular}{|c|c|c|c|c|c|c|c|c|c|c|c|c|c|c|c|}
\hline STUDIES & ITEM 1 & ITEM 2 & ITEM 3 & ITEM 4 & ITEM 5 & ITEM 6 & ITEM 7 & ITEM 8 & ITEM 9 & ITEM 10 & ITEM 11 & ITEM 12 & ITEM 13 & ITEM 14 & TOT YES \\
\hline Batcho 2012 & $Y$ & $\mathrm{Y}$ & N.R.* & Y & N.R.* & $\mathrm{N}$ & $\mathrm{N}$ & N.A.** & $\mathrm{Y}$ & N.R.* & Y & $\mathrm{N}$ & N.A. ${ }^{* *}$ & Y & 6 \\
\hline Brosseau 1996 & $Y$ & $\mathrm{Y}$ & Y & Y & N.R.* & $\mathrm{N}$ & $\mathrm{N}$ & Y & $\mathrm{Y}$ & N.R.* & $Y$ & $\mathrm{~N}$ & N.A.** & Y & 8 \\
\hline Chan 2006 & $\mathrm{Y}$ & $\mathrm{N}$ & N.R.* & $\mathrm{Y}$ & N.R.* & $\mathrm{N}$ & $\mathrm{N}$ & Y & $\mathrm{Y}$ & Y & $Y$ & $\mathrm{~N}$ & Y & Y & 8 \\
\hline Chen 2013 & $\mathrm{Y}$ & $\mathrm{Y}$ & N.R.* & $\mathrm{Y}$ & N.R.* & $\mathrm{N}$ & $\mathrm{N}$ & $\mathrm{N}$ & $\mathrm{Y}$ & $\mathrm{N}$ & $Y$ & $\mathrm{Y}$ & N.A.** & N.R.* & 6 \\
\hline Cup 2003 & $\mathrm{Y}$ & $\mathrm{Y}$ & N.R.* & Y & N.R.* & $\mathrm{N}$ & $\mathrm{N}$ & $\mathrm{N}$ & $\mathrm{Y}$ & $\mathrm{Y}$ & $Y$ & $\mathrm{~N}$ & N.A.** & Y & 7 \\
\hline Drummond 2000 & $\mathrm{Y}$ & $\mathrm{N}$ & Y & N.R.* & N.R.* & $\mathrm{N}$ & $\mathrm{N}$ & $\mathrm{N}$ & $\mathrm{N}$ & $\mathrm{Y}$ & $\mathrm{N}$ & N.A.** & $\mathrm{N}$ & Y & 4 \\
\hline Fallahpour 2011 & $\mathrm{Y}$ & $Y$ & $Y$ & Y & N.R.* & $\mathrm{N}$ & $\mathrm{N}$ & N.R.* & $Y$ & $\mathrm{~N}$ & $Y$ & $\mathrm{~N}$ & N.A. ${ }^{* *}$ & N.R.* & 6 \\
\hline Fallahpour 2011-2 & $\mathrm{Y}$ & $Y$ & Y & $Y$ & N.R.* & $\mathrm{N}$ & $\mathrm{N}$ & N.R.* & $Y$ & $\mathrm{~N}$ & $Y$ & N.R.* & N.A. ${ }^{* *}$ & Y & 7 \\
\hline Gompertz 1994 & $\mathrm{Y}$ & $Y$ & N.R.* & $Y$ & N.R.* & $\mathrm{N}$ & $\mathrm{N}$ & $\mathrm{N}$ & $Y$ & $Y$ & $Y$ & N.R.* & $\mathrm{N}$ & Y & 7 \\
\hline Hamed 2012 & $\mathrm{Y}$ & $Y$ & N.R.* & $Y$ & N.R.* & $\mathrm{N}$ & $\mathrm{N}$ & $\mathrm{Y}$ & $\mathrm{Y}$ & $\mathrm{N}$ & $Y$ & N.R.* & N.A. ${ }^{* *}$ & N.R.* & 6 \\
\hline Horgan 2006 & Y & $\mathrm{N}$ & N.R.* & $Y$ & N.R.* & $\mathrm{N}$ & $\mathrm{N}$ & $\mathrm{N}$ & $Y$ & $\mathrm{~N}$ & $Y$ & $\mathrm{~N}$ & N.A.** & N.R.* & 4 \\
\hline Hsueh 2012 & $\mathrm{Y}$ & $\mathrm{N}$ & N.R.* & $\mathrm{Y}$ & N.R.* & $\mathrm{N}$ & $\mathrm{N}$ & Y & $\mathrm{Y}$ & $\mathrm{Y}$ & $\mathrm{Y}$ & $\mathrm{N}$ & N.R.* & N.R.* & 6 \\
\hline Jansa 2004 & $Y$ & $\mathrm{~N}$ & N.R.* & Y & N.R.* & $\mathrm{N}$ & $\mathrm{N}$ & $\mathrm{N}$ & $\mathrm{Y}$ & Y & $Y$ & N.R.* & N.R.* & N.R.* ${ }^{*}$ & 5 \\
\hline Katz 2003 & $\mathrm{Y}$ & $\mathrm{Y}$ & N.R.* & $\mathrm{N}$ & N.R.* & $\mathrm{N}$ & $\mathrm{N}$ & Y & $\mathrm{Y}$ & $\mathrm{Y}$ & $Y$ & N.R.* & Y & N.R.* & 7 \\
\hline Korpelainen 1997 & $\mathrm{Y}$ & $\mathrm{Y}$ & N.R.* & $\mathrm{Y}$ & N.R.* & $\mathrm{N}$ & $\mathrm{N}$ & $\mathrm{N}$ & $\mathrm{Y}$ & $\mathrm{Y}$ & $Y$ & N.R.* & $\mathrm{N}$ & N.R.* & 6 \\
\hline Lee2014 & $\mathrm{Y}$ & $\mathrm{N}$ & N.R.* & $\mathrm{Y}$ & N.R.* & $\mathrm{N}$ & $\mathrm{N}$ & $\mathrm{N}$ & Y & Y & $Y$ & $\mathrm{~N}$ & N.R.* & N.R.* & 5 \\
\hline Leung 2007 & $\mathrm{Y}$ & $\mathrm{N}$ & N.R.* & $\mathrm{Y}$ & N.R.* & $\mathrm{N}$ & $\mathrm{N}$ & $\mathrm{N}$ & $\mathrm{Y}$ & $\mathrm{Y}$ & $\mathrm{Y}$ & $\mathrm{N}$ & N.A.** & Y & 6 \\
\hline Li 2016 & $\mathrm{Y}$ & $Y$ & Y & $Y$ & N.R.* & $\mathrm{N}$ & $\mathrm{N}$ & $\mathrm{N}$ & $\mathrm{Y}$ & $\mathrm{N}$ & Y & $\mathrm{N}$ & Y & Y & 8 \\
\hline Lin 2012 & $\mathrm{Y}$ & $\mathrm{N}$ & $\mathrm{N}$ & $\mathrm{Y}$ & N.R.* & $\mathrm{N}$ & $\mathrm{N}$ & $\mathrm{N}$ & $\mathrm{Y}$ & Y & $Y$ & N.R.* & N.R.* & Y & 6 \\
\hline Lincoln 1990 & Y & $\mathrm{N}$ & N.R.* ${ }^{*}$ & Y & N.R.* & $\mathrm{N}$ & $\mathrm{N}$ & $\mathrm{N}$ & $\mathrm{Y}$ & $\mathrm{N}$ & Y & N.R.* & N.R.* ${ }^{*}$ & N.R.* ${ }^{*}$ & 4 \\
\hline Lincoln 1992 & Y & Y & Y & N.R.* & N.R.* & $\mathrm{N}$ & $\mathrm{N}$ & N.R.* & $\mathrm{Y}$ & $\mathrm{N}$ & Y & N.R.* & $\mathrm{N}$ & N.R.* & 5 \\
\hline Lundgren-Nilsson 2006 & Y & $\mathrm{N}$ & N.R.* & N.R.* & N.R.* & $\mathrm{N}$ & $\mathrm{N}$ & N.R.* & $\mathrm{Y}$ & $\mathrm{N}$ & Y & N.R.* & N.A.** & N.R.* & 3 \\
\hline Maujean 2013 & Y & $\mathrm{Y}$ & $\mathrm{N}$ & Y & N.R.* & $\mathrm{N}$ & $\mathrm{N}$ & N.R.* & $\mathrm{Y}$ & $\mathrm{N}$ & $Y$ & N.R.* & N.A.** & N.R.* & 5 \\
\hline Mennem 2012 & $Y$ & $\mathrm{~N}$ & N.R.* & $\mathrm{Y}$ & N.R.* & $\mathrm{N}$ & $\mathrm{N}$ & N.R.* & $\mathrm{Y}$ & $\mathrm{N}$ & $Y$ & N.R.* & N.A.** & N.R.* & 4 \\
\hline Miki 2015 & $\mathrm{Y}$ & $Y$ & N.R.* & $\mathrm{Y}$ & N.R.* & $\mathrm{N}$ & $\mathrm{N}$ & $\mathrm{N}$ & $\mathrm{Y}$ & $\mathrm{Y}$ & $Y$ & $\mathrm{~N}$ & N.A.** & N.R.* & 6 \\
\hline Monteiro 2017 & $\mathrm{Y}$ & $Y$ & Y & $Y$ & N.R.* & $\mathrm{N}$ & $\mathrm{N}$ & N.R.* & $Y$ & Y & $Y$ & N.R.* & N.R.* & Y & 8 \\
\hline Naghdi 2015 & $\mathrm{Y}$ & $\mathrm{N}$ & N.R.* & Y & N.R.* & $\mathrm{N}$ & $\mathrm{N}$ & N.R.* & $Y$ & Y & $Y$ & $\mathrm{~N}$ & N.R.* & Y & 6 \\
\hline Ottiger 2015 & $\mathrm{Y}$ & $\mathrm{Y}$ & N.R.* & N.R.* & N.R.* & $\mathrm{N}$ & $\mathrm{N}$ & N.R.* & $Y$ & Y & $Y$ & N.R.* & N.R.* & N.R.* & 5 \\
\hline Pang 2011 & $Y$ & $\mathrm{~N}$ & N.R.* & Y & N.R.* & $\mathrm{N}$ & $\mathrm{N}$ & N.A.** & $Y$ & Y & $Y$ & N.R.* & Y & N.R.* & 6 \\
\hline Piercy 2000 & $Y$ & $Y$ & $\mathrm{~N}$ & $Y$ & N.R.* & $\mathrm{N}$ & $\mathrm{N}$ & N.R.* ${ }^{*}$ & $Y$ & $\mathrm{~N}$ & $Y$ & Y & N.A. ${ }^{* *}$ & N.R.* ${ }^{*}$ & 6 \\
\hline Post 2003 & $Y$ & $\mathrm{~N}$ & N.R.* & $\mathrm{Y}$ & N.R.* & $\mathrm{N}$ & $\mathrm{N}$ & N.R.* & $\mathrm{Y}$ & Y & Y & N.R.* & N.R.* & N.R.* & 5 \\
\hline Schlote 2009 & Y & $\mathrm{N}$ & Y & Y & N.R.* & $\mathrm{N}$ & $\mathrm{N}$ & N.R.* & $\mathrm{Y}$ & Y & Y & N.R.* & $\mathrm{N}$ & N.R.* ${ }^{*}$ & 6 \\
\hline Spencer 1986 & Y & $\mathrm{N}$ & N.R.* & N.R.* & N.R.* & $\mathrm{N}$ & $\mathrm{N}$ & N.R.* & Y & N.R.* & Y & N.R.* & N.R.* & N.R.* & 3 \\
\hline Teixeira-Salmela 2007 & Y & $\mathrm{N}$ & N.R.* & Y & Y & $\mathrm{N}$ & $\mathrm{N}$ & Y & $\mathrm{Y}$ & Y & Y & N.R.* & $\mathrm{N}$ & Y & 8 \\
\hline Tooth 2003 & Y & $\mathrm{Y}$ & Y & $\mathrm{Y}$ & N.R.* & $\mathrm{N}$ & $\mathrm{N}$ & $\mathrm{N}$ & Y & Y & Y & N.R. ${ }^{*}$ & N.R.* & N.R.* & 7 \\
\hline Uswatte 2006 & Y & $\mathrm{N}$ & N.R.* & $\mathrm{Y}$ & N.R.* & $\mathrm{N}$ & $\mathrm{N}$ & N.R.* & $\mathrm{Y}$ & Y & Y & Y & N.R.* & N.R.* & 6 \\
\hline Van der Lee 2004 & $\mathrm{Y}$ & $\mathrm{N}$ & Y & $\mathrm{Y}$ & N.R.* & $\mathrm{N}$ & $\mathrm{N}$ & $\mathrm{N}$ & Y & Y & $Y$ & N.R.* & $Y$ & N.R.* & 7 \\
\hline Wang 2011 & $\mathrm{Y}$ & $Y$ & N.R.* & $Y$ & Y & $\mathrm{N}$ & $\mathrm{N}$ & $Y$ & $\mathrm{Y}$ & Y & Y & N.R.* & $\mathrm{Y}$ & N.R.* & 9 \\
\hline Yamada 2006 & $\mathrm{Y}$ & $\mathrm{Y}$ & $\mathrm{N}$ & $\mathrm{N}$ & N.R.* & $\mathrm{N}$ & $\mathrm{N}$ & $Y$ & $Y$ & $Y$ & $Y$ & N.R.* & N.R.* & N.R.* & 6 \\
\hline Total YES & 39 & 20 & 10 & 32 & 2 & 0 & 0 & 8 & 38 & 24 & 38 & 3 & 6 & 13 & \\
\hline
\end{tabular}

${ }^{*}$ N.R. Not Reported.

**N.A. Not Applicable.

the variables of interest as gold standards and they were defined in detail. Only one article ${ }^{29}$ did not clarify the comparative measurements. Just over half, 24, of the studies provided the exposure for each person measured more than once during the course of the study period. In 3 studies, ${ }^{25,26,57}$ this information was not reported. Only 3 studies ${ }^{28,54,60}$ provided sufficient information to determine they were blinded.

\section{Follow-up rate; statistical analyses}

Thirteen studies ${ }^{25-27,29,30,32,33,41-43,50,51,58}$ reported data on follow-up. Usually, an acceptable overall follow-up rate is considered to be $80 \%$ or more of participants whose exposures were measured at baseline. In 6 studies, ${ }^{27,38,40,42,54}$ this percentage was lower than $20 \%$. Only in $13^{25-27,29,30,32,33,41-43,50,51,58}$ studies were key potential confounding variables measured and adjusted for, such as by statistical adjustment for baseline differences; in other studies this process was not reported.

\section{Outcome measures}

The two most commonly used stroke scales resulted from this review are the Frenchay Activities Index $(\mathrm{FAI})^{42,50,54,55}$ and the FIM $^{26,51}$ with two modified versions. ${ }^{46,49}$ The FAI is a formal interview for patients who have suffered a stroke or their caregivers to compare functional abilities preceding and following a stroke. It has been used to assess ADLs three and six months before a stroke and consists of 15 items that measure complex activities in the categories of domestic activity, work/leisure, and outdoor activity. ${ }^{50}$ The FAI score is based on the frequency with which activities are performed, ranging from 0 (inactive) to 45 (very active): $0-15=$ inactive, $16-30=$ moderately active, and $31-45=$ very active. The cutoff of $\geq 18$ has been used as a predictor of mild disability after stroke. ${ }^{50}$ According to its developer, the FAI can be used to record premorbid levels of functioning, thus facilitating the negotiation of realistic rehabilitation goals, and to measure changes in the levels of functioning due to stroke. ${ }^{55}$ It usually takes only a few minutes to complete and is very easy to administer. ${ }^{43}$ The FAI has shown significant correlations with the Barthel Index, the Sickness Impact Profile, and the Stroke Impact Scale-ADL/IADL, as well as with the modified Nottingham Extended ADL. ${ }^{50}$

The $\mathrm{FlM}^{26,51}$ is widely used throughout the world; it provides a uniform system of measurement for disability based 
on the International Classification of Functioning, Disability and Health. ${ }^{64}$ It measures the level of a patient's disability and indicates how much assistance is required for the individual to carry out ADLs through 18 items composed of 13 motor tasks and 5 cognitive tasks. ${ }^{26}$ The Scandinavian study ${ }^{46}$ explored in this review validated only the motor subscale of the FIM. The Japanese study ${ }^{49}$ validated the FIM in association with the FAM. The FAM is a 30 -item global measure of disability that was constructed by adding the 12 unique items [that emphasize the cognitive, communicative, and psychosocial aspects of the disability) to the original 18 items of the FIM; it was developed as a measure for evaluating disability in patients with traumatic brain injury (TBI). Items of the FIM +FAM have been grouped into two subscales based on principal component analysis: a motor subscale comprised of 16 items and a cognitive subscale comprised of 14 items. ${ }^{49}$ Both the FIM and the FAM use a 7-point scoring system based on whether or not the assistance of another person is required for an individual to perform ADLs effectively. A rating of 1 indicates total dependence and 7 complete independence, with intermediate levels rated as: 6 modified independence, 5 supervision or set up, 4 minimal contact assistance or the subject expends $>75 \%$ of the effort, 3 moderate assistance or the subject expends 50 to $74 \%$ of the effort, and 2 maximal assistance or the subject expends 25 to $49 \%$ of the effort. ${ }^{51}$ It is recommended that the FIM be administered by welltrained raters. ${ }^{49}$

\section{Discussion}

This study was conducted by a research group composed by medical doctors and rehabilitation professionals from the "Sapienza" University of Rome and from "Rehabilitation \& Outcome Measure Assessment" (R.O.M.A.) association. R.O. M.A. association in the last few years has dealt with several systematic reviews and the validation of many outcome measures in Italy. ${ }^{65-74}$

\section{Summary of the quality of the evidence}

The assessment of ADLs in adults who have had a stroke is a crucial moment for the entire field of rehabilitation. Outcome measures are frequently used to determine if patients have made meaningful changes in their recovery process and may influence the intensity and duration of care. Researchers use outcome measures during the investigation of the efficacy and effectiveness of a given treatment intervention. Therefore, the aim of this study was to provide clinicians and researchers with evidence-based recommendations regarding what outcome measures should be used to assess the loss of functionality in the ADLs of patients following a stroke. This was achieved by reviewing the most frequently used outcome measures and determining whether there is evidence to support their use. The data available in the major databases up to July 2017 permit the identification of different measurement tools internationally. The research was conducted using keywords and no time limits were set so as not to exclude any study that could have made important contributions to the review. The studies that emerged were published between November $1986^{57}$ and March. $2017^{50}$ In total, 33 studies tracked the use of an assessment tool. ${ }^{25-63}$ In these studies, a strong heterogeneity of validated tools among the various national contexts can be seen. This heterogeneity can be assumed to have a positive meaning if one thinks about the multiple needs of the clinical context, but certainly leads to the need to make the tools more suitable for various cultural contexts. These findings suggest that clinicians have conflicting or incomplete information available to use when making decisions in patient care; furthermore, the lack of consistency and the deficiency of standardization in outcome assessment has hindered comparative research and metaanalysis. Further investigation of outcome measures would benefit patients, researchers, and clinicians. A universal, validated outcome measure is needed to allow comparisons across practice; therefore, we recommend that future researchers use a common set of outcome assessments.

The variety of methods used throughout the literature to measure responsiveness illustrates the present problem of defining and standardizing a method or standard descriptor that can be used to accurately report responsiveness among various outcome measures. The COSMIN checklist was published in 2010 to assess the methodological quality of studies on measurement properties. ${ }^{20-23}$ Through an international consensus process, the COSMIN framework was developed, providing specific recommendations on terminology, taxonomy, and methodology in studies dealing with PROMs and their measurement properties. We performed this systematic review in line with the recommendations of the COSMIN initiative but we found great difficulty in tracking all the desired values; for some studies, this trend was hypothetically attributed to the year of publication. The assessment of the studies through the QAT provided a more specific overview of the quality of the studies tracked by the review. Only $16^{25,28,31,32,34,36,42,43,47-53,62}$ of the 39 studies were published after the publication of the COSMIN checklist and these guidelines were not followed in the majority of the included studies; however, according to the QAT, the studies published after 2010 do seem to have higher quality.

This review has demonstrated that no perfect stroke-ADL scale exists, therefore many assessment tools, spanning various ADL domains, are available to clinicians and researchers working with stroke survivors. We have described the flavor of the marked heterogeneity in the use of assessment tools and we have deliberately avoided suggestions that one scale is better than another. We have focused, as exemplars, on the two most commonly used stroke scales: the Frenchay Activities Index (FAI $)^{42,50,54,55}$ and the FIM $^{26,51}$ with two modified versions. ${ }^{46,49}$ These scales have been validated, are familiar to many, and have proven utility with each suited to differing assessment scenarios. Thus, in the absence of a "perfect" assessment, we recommend continuing the use, validation, and adaptation of these assessment scales for adults with stroke in different countries to develop a common language throughout the world for discussing ADLs and functionality.

In response to the secondary objective of this review, it was not possible to find tools to assess the loss of functionality in 
ADLs specific to adults with stroke that were validated in the Italian context.

\section{Limitations of the study}

There are several limitations of this review that need to be considered. Despite having systematically searched three electronic databases, it is possible that not all relevant studies were identified. Studies may have been published in journals that were not covered by the databases. In addition, this review only included published studies; therefore, studies that have been submitted and not accepted for publication or were accepted for publication only recently would be excluded. Only Englishlanguage articles were included, making it possible that this systematic review is not a complete representation of the evidence available worldwide. Finally, studies may not have been identified with the search strategy used, in fact some instruments, such as SIS 3.0 and others, are not considered in the study. Due to the nature of problems with ADLs, which affect many areas of life, it is possible that some relevant articles did not use the words applied in the search strategy; for example, if an article was about the validation of an outcome measure for assessing disability, it would not have been included.

Finally, one of the bias associated with the FAI and FIM is their publication date and the fact that they have existed for much longer, especially given they were the only ones available at their respective times. As a result, they have grown to prominence and have been revised more over time to present with better psychometric properties.This systematic review revealed large inconsistencies among the current available ADL outcome measures. The limitations of this study can be attributed to the difficulty of bringing the reporting of studies according to the general principles; this makes it difficult to compare study results and to create norms and standards for the loss of functionality in the ADLs of stroke patients. We believe that further exploration, analysis, and adaptation of existing instruments is needed rather than the development of new outcome measures.

\section{Conclusions}

To our knowledge, this is the first systematic review that describes the outcome measures of the loss of functionality in the ADLs of adults with stroke with the parameters of validity, reliability, responsiveness, and languages in the literature. It is important for the development of clinical practice and research that practical and appropriate measures are universally accepted; this would allow comparisons and metaanalysis of high quality randomized controlled trials of people with this ever-increasing injury. At present, there is no one broadly accepted ADL assessment tool for adults with stroke that would allow for the comparison of study results. It is hoped that this review has emphasized the need for agreement among researchers as to which tool must be studied in depth or adapted to other national contexts as well as which one measurement instrument should be standardized in its use in order to develop universal norms and standards for the performance of adults with stroke. Thanks to this review, we have highlighted the lack of a valid and reliable assessment tool for the ADL functionality of adults with stroke in Italy. Future studies could enrich and integrate the Italian rehabilitation context of adults with stroke by culturally adapting one of the tools that emerged from this review to create standardized and shared evaluation paths.

\section{ORCID}

Galeoto Giovanni (D) http://orcid.org/0000-0002-9043-5686

\section{References}

1. Stroke Unit Trialists' Collaboration. Organised inpatient (stroke unit) care for stroke. Cochrane Database Syst Rev. 2007;4: CD000197.

2. Wardlaw JM, Murray V, Berge E, Del Zoppo GJ. Thrombolysis for acute ischaemic stroke. Cochrane Database Syst Rev. 2009;4: CD000213.

3. Sandercock PAG, Counsell C, Kamal AK. Anticoagulants for acute ischaemic stroke. Cochrane Database Syst Rev. 2008;4:CD000024.

4. MedicineNet: https://www.medicinenet.com/script/main/hp. asp. updated 18 August; Accessed September 2017.

5. Harrison JK, McArthur KS, Quinn TJ. Assessment scales in stroke: clinimetric and clinical considerations. Clin Interv Aging. 2013;8:201-211. doi:10.2147/CIA.S32405

6. Oxman AD, Cook DJ, Guyatt GH. Users' guides to the medical literature. VI. How to use an overview. Evidence-based medicine working group. JAMA. 1994;272:1367-1371. doi:10.1001/ jama.1994.03520170077040

7. Swingler GH, Volmink J, Ioannidis JP. Number of published systematic reviews and global burden of disease: database analysis. BMJ. 2003;327:1083-1084. doi:10.1136/bmj.327.7420.895

8. Higgins JPT, Green S Cochrane handbook for systematic reviews of interventions: the cochrane collaboration; 2011. http://handbook. cochrane.org/. updated March; Accessed December 18, 2013.

9. Higgins JPT, Lasserson T, Chandler J, Tovey D, Churchill R. Methodological Expectations of Cochrane Intervention Reviews (MECIR) standards for the conduct and reporting of new cochrane intervention reviews, reporting of protocols and the planning, conduct and reporting of updates. https://methods.cochrane.org/ mecir. updated 18 August; Accessed September 2013.

10. Chandler J, Churchill R, Higgins J, Lasserson T, Tovey D Methodological standards for the conduct of new cochrane intervention reviews version 2.3: the cochrane library; 2013. http:// editorial-unit.cochrane.org/sites/editorialunit.cochrane.org/files/ uploads/MECIR_conduct_standards\%202.3\%2002122013.pdf. updated 2 December; Accessed October 21, 2014.

11. Methods guide for effectiveness and comparative effectiveness reviews. Rockville MD: Agency for Healthcare Research and Quality; 2008. http://www.ncbi.nlm.nih.gov/books/NBK47095/. Accessed December 18, 2013.

12. Moher D, Liberati A, Tetzlaff J, Altman DG, The PRISMA Group. Preferred reporting items for systematic reviews and meta-analyses: the PRISMA statement. PLoS Med. 2009;6(7):e1000097. doi:10.1371/journal.pmed.1000097

13. Liberati A, Altman DG, Tetzlaff J, et al. The PRISMA statement for reporting systematic reviews and meta-analyses of studies that evaluate health care interventions: explanation and elaboration. PLoS Med. 2009;6(7):e1000100. doi:10.1371/journal.pmed.1000100

14. Rethlefsen ML, MLS, Koffel J, Kirtley S. PRISMA-search (PRISMA-S) extension to PRISMA development protocol. http://www.equator-net work.org/wp-content/uploads/2009/02/Protocol-PRISMA-S-Delphi. pdf. updated 18 august; Accessed September 2013.

15. The Internet Stroke Centre. Stroke assessment scales overview. 1995. http://www.strokecenter.org/trials/scales/scales-overview.htm. Accessed December 7, 2012.

16. Wade DT. Measurement in Neurological Rehabilitation. Oxford: Oxford University Press; 1992. 
17. Fava GA, Tomba E, Sonino N. Clinimetrics: the science of clinical measurements. Int J Clin Pract. 2012;66:11-15. doi:10.1111/ ijcp. 12013

18. Noyes J, Lewin S. Chapter 5: extracting qualitative evidence. In: Noyes J, Booth A, Hannes K, et al., eds. Supplementary Guidance for Inclusion of Qualitative Research in Cochrane Systematic Reviews of Interventions. Version 1. Cochrane Collaboration Qualitative Methods Group; 2011. http://cqrmg.cochrane.org/sup plemental-handbook-guidance. Accessed August, 2011

19. Bates S, Coren E. Systematic Map No.1: The Extent and Impact of Parental Mental Health Problems on Families and the Acceptability, Accessibility and Effectiveness of Interventions. London: SCIE; 2006.

20. Mokkink LB, Terwee CB, Patrick DL, et al. The COSMIN study reached international consensus on taxonomy, terminology, and definitions of measurement properties for health-related patient-reported outcomes. J Clin Epidemiol. 2010;63(7):737-745. doi:10.1016/j.jclinepi.2010.02.006

21. Mokkink LB, Terwee CB, Patrick DL, et al. The COSMIN checklist for assessing the methodological quality of studies on measurement properties of health status measurement instruments: an international Delphi study. Qual Life Res. 2010;19(4):539-549. doi:10.1007/s11136-0109655-z

22. Mokkink LB, Terwee CB, Knol DL, et al. The COSMIN checklist for evaluating the methodological quality of studies on measurement properties: a clarification of its content. BMC Med Res Methodol. 2010;10:22. doi:10.1186/1471-2288-10-22

23. Mokkink LB, Terwee CB, Patrick DL, et al. COSMIN checklist manual. COSMIN; 2012. http://www.cosmin.nl/images/upload/ files/COSMIN\%20checklist\%20manual\%20v9.pdf.

24. Shuang F, Hou SX, Z JL, Ren DF, Cao Z, Tang JG. 2014. Accessed October 13, 2017 (GMT). doi:10.1371/journal.pone.0111695.t001.

25. Batcho CS, Tennant A, Thonnard JL. ACTIVLIM-stroke: a crosscultural Rasch-built scale of activity limitations in patients with stroke. Stroke. 2012;43(3):815-823. doi:10.1161/ STROKEAHA.111.638965

26. Brosseau L, Potvin L, Philippe P, Boulanger YL, Dutil E. The construct validity of the functional independence measure as applied to stroke patients. Physiother Theory Pract. 1996;12 (3):161-171. doi:10.3109/09593989609036432

27. Chan VWK, Chung JCC, Packer TL. Validity and reliability of the activity card sort-Hong Kong version. OTJR-Occup Part Heal. 2006;26(4):152-158. doi:10.1177/153944920602600405

28. Chen HF, Wu CY, Lin KC, et al. Rasch validation of a combined measure of basic and extended daily life functioning after stroke. Neurorehabil Neural Repair. 2013;27(2):125-132. doi:10.1177/ 1545968312457828

29. Cup EH, Scholte Op Reimer WJ, Thijssen MC, van Kuyk-Minis MA. Reliability and validity of the Canadian occupational performance measure in stroke patients. Clin Rehabil. 2003;17 (4):402-409. doi:10.1191/0269215503cr635oa

30. Drummond AE, Parker CJ, Gladman JR, Logan PA, TOTAL STUdY Group. Development and validation of the Nottingham Leisure Questionnaire (NLQ). Clin Rehabil. 2001;15(6):647-656. doi:10.1191/0269215501cr438oa

31. Fallahpour M, Jonsson H, Joghataei MT, Kottorp A. Impact on Participation and Autonomy (IPA): psychometric evaluation of the Persian version to use for persons with stroke. Scand J Occup Ther. 2011;18:59-71. doi:10.3109/11038121003628353

32. Fallahpour M, Tham K, Joghataei MT, Eriksson G, Jonsson H. Occupational gaps in everyday life after stroke and the relation to functioning and perceived life satisfaction. OTJR. 2011;31(4):200208.

33. Gompertz P, Pound P, Ebrahim S. Validity of the extended activities of daily living scale. Clin Rehabil. 1994;8(4):275-280. doi:10.1177/026921559400800401

34. Hamed R, Tariah HA, Malkawi S, Holm MB. The Arabic version of the Mayo-Portland adaptability inventory 4: a validation study. Int J Rehabil Res. 2012;35(3):243-247. doi:10.1097/MRR.0b013e3283544c9f
35. Horgan NF, Cunningham CJ, Coakley D, Walsh JB, O'Regan M, Finn AM. The stroke activity scale: results of a validity study. Disabil Rehabil. 2006;28(15):937-941. doi:10.1080/09638280500404461

36. Hsueh IP, Wang CH, Liou TH, Lin CH, Hsieh CL. Test-retest reliability and validity of the comprehensive activities of daily living measure in patients with stroke. J Rehabil Med. 2012;44:637-641. doi:10.2340/16501977-0915

37. Jansa J, Pogacnik T, Gompertz P. An evaluation of the extended Barthel index with acute ischemic stroke patients. Neurorehabil Neural Repair. 2004;18(1):37-41. doi:10.1177/0888439003262287

38. Katz N, Karpin H, Lak A, Furman T, Hartman-Maeir A. Participation in occupational performance: reliability and validity of the activity card sort. OTJR. 2003;23:10-17.

39. Korpelainen JT, Niilekselä E, Myllylä VV. The Sunnaas index of activities of daily living: responsiveness and concurrent validity in stroke. Scand J Occup Ther. 1997;4:31-36. doi:10.3109/ 11038129709035719

40. Lee YC, Chen SS, Koh CL, Hsueh IP, Yao KP, Hsieh CL. Development of two barthel index-based supplementary scales for patients with stroke. PLoS One. 2014;9(10):e110494. doi:10.1371/ journal.pone.0110494

41. Leung SO, Chan CC, Shah S. Development of a Chinese version of the modified Barthel Index - validity and reliability. Clin Rehabil. 2007;21(10):912-922. doi:10.1177/0269215507077286

42. Li HY, Cheng HL, Fang L, Bi RX, Fang XQ, Hu M. Validity and reliability of the Chinese version of the daily living self-efficacy scale among stroke patients. Int J Rehabil Res. 2016;39(3):219-225. doi:10.1097/MRR.0000000000000170

43. Lin KC, Chen HF, Wu CY, Yu TY, Ouyang P. Multidimensional Rasch validation of the Frenchay activities index in stroke patients receiving rehabilitation. J Rehabil Med. 2012;44(1):58-64. doi:10.2340/16501977-0911

44. Lincoln NB, Edmans JA. A re-validation of the Rivermead ADL scale for elderly patients with stroke. Age Ageing. 1990;19:19-24.

45. Lincoln NB, Gladman JR. The extended activities of daily living scale: a further validation. Disabil Rehabil. 1992;14:41-43.

46. Lundgren-Nilsson A, Tennant A, Grimby G, Sunnerhagen KS. Cross-diagnostic validity in a generic instrument: an example from the functional independence measure in Scandinavia. Health Qual Life Outcomes. 2006;4:55. doi:10.1186/1477-75254-55

47. Maujean A, Davis P, Kendall E, Casey L, Loxton N. The daily living self-efficacy scale: a new measure for assessing self-efficacy in stroke survivors. Disabil Rehabil. 2014;36(6):504-511. doi:10.3109/ 09638288.2013.804592

48. Mennem TA, Warren M, Yuen HK. Preliminary validation of a vision-dependent activities of daily living instrument on adults with homonymous hemianopia. Am J Occup Ther. 2012;66 (4):478-482. doi:10.5014/ajot.2012.004762

49. Miki E, Yamane S, Yamaoka M, et al. Validity and reliability of the Japanese version of the FIM + FAM in patients with cerebrovascular accident. Scand J Occup Ther. 2016;23(5):398-404. doi:10.3109/11038128.2015.1095236

50. Monteiro M, Maso I, Sasaki AC, Neto BN, Filho OJ, Pinto EB. Validation of the Frenchay activity index on stroke victims. Arq Neuropsiquiatr. 2017;75(3):167-171. doi:10.1590/0004-282X20170014

51. Naghdi S, Ansari NN, Raji P, Shamili A, Amini M, Hasson S. Cross-cultural validation of the Persian version of the functional independence measure for patients with stroke. Disabil Rehabil. 2016;38(3):289-298. doi:10.3109/09638288.2015.1036173

52. Ottiger B, Vanbellingen T, Gabriel C, et al. Validation of the new Lucerne ICF Based Multidisciplinary Observation Scale (LIMOS) for stroke patients. PLoS One. 2015;10(6):e0130925. doi:10.1371/ journal.pone.0130925

53. Pang MY, Lau RW, Yeung PK, Liao LR, Chung RC. Development and validation of the Chinese version of the reintegration to normal living index for use with stroke patients. J Rehabil Med. 2011 Feb;43(3):243-250. doi:10.2340/16501977-0660 
54. Piercy M, Carter J, Mant J, Wade DT. Inter-rater reliability of the Frenchay activities index in patients with stroke and their carers. Clin Rehabil. 2000 Aug;14(4):433-440. doi:10.1191/0269215500cr327oa

55. Post MW, de Witte LP. Good inter-rater reliability of the Frenchay activities index in stroke patients. Clin Rehabil. 2003;17 (5):548-552. doi:10.1191/0269215503cr648oa

56. Schlote A, Richter M, Wunderlich MT, et al. WHODAS II with people after stroke and their relatives. Disabil Rehabil. 2009;31 (11):855-864. doi:10.1080/09638280802355262

57. Spencer C, Clark M, Smith DS. A modification of the Northwick Park ADL index (The Australian ADL index). Br J Occup Ther. 1986;49(11):350-353. doi:10.1177/030802268604901103

58. Teixeira-Salmela LF, Devaraj R, Olney SJ. Validation of the human activity profile in stroke: A comparison of observed, proxy and self-reported scores. Disabil Rehabil. 2007;29(19):1518-1524. doi:10.1080/09638280601055733

59. Tooth LR, McKenna KT, Smith M, O’Rourke PK. Reliability of scores between stroke patients and significant others on the Reintegration to Normal Living (RNL) index. Disabil Rehabil. 2003;25(9):433-440. doi:10.1080/0963828031000069726

60. Uswatte G, Taub E, Morris D, Light K, Thompson PA. The motor activity log-28: assessing daily use of the hemiparetic arm after stroke. Neurology. 2006;67(7):1189-1194. doi:10.1212/ 01.wnl.0000238164.90657.c2

61. Van der Lee JH, Beckerman H, Knol DL, de Vet HC, Bouter LM. Clinimetric properties of the motor activity log for the assessment of arm use in hemiparetic patients. Stroke. 2004;35(6):1410-1414 doi:10.1161/01.STR.0000126900.24964.7e

62. Wang TN, Lin KC, Wu CY, Chung CY, Pei YC, Teng YK. Validity, responsiveness and clinical important difference of the ABILHAND questionnaire in patient with stroke. Arch Phys Med Rehabil. 2011;92(7):1086-1091. doi:10.1016/j.apmr.2011.01.020

63. Yamada S, Liu M, Hase K, et al. Development of a short version of the motor fim for use in long-term care settings. J Rehabil Med. 2006;38:50-56

64. World Health Organization Geneva. Towards a common language for functioning, disability and health ICF. WHO/EIP/GPE/CAS/
01.3; 2002. http://www.who.int/classifications/icf/icfchecklist.pdf? $\mathrm{ua}=1$.

65. Galeoto G, Lauta A, Palumbo A, Castiglia SF, Mollica R, Santilli V. The Barthel index: Italian translation, adaptation and validation. Int J Neurol Neurother. 2015;2:1-7.

66. Culicchia G, Nobilia M, Asturi M, et al. Cross-cultural adaptation and validation of the Jebsen-Taylor hand function test in an Italian population. Rehabilitation Research and Practice. 2016; Article ID 8970917:12.

67. Castiglia SF, Galeoto G, Lauta A, et al. The culturally adapted Italian version of the Barthel Index (IcaBI): assessment of structural validity, inter-rater reliability and responsiveness to clinically relevant improvements in patients admitted to inpatient rehabilitation centers. Funct Neurol. 2017;32(4):221. doi:10.11138/FNeur/2017.32.4.221

68. Marquez MA, De Santis R, Ammendola V, et al. Cross-cultural adaptation and validation of the "spinal cord injury-falls concern scale" in the Italian population. Spinal Cord. 2018;1:712-718

69. Galeoto G, Sansoni J, Scuccimarri M, et al. A psychometric properties evaluation of the Italian version of the geriatric depression scale. Depress Res Treat. 2018. doi:10.1155/2018/1797536

70. Murgia M, Bernetti A, Delicata $\mathrm{M}$, et al. Inter-and intra-interviewer reliability of Italian version of pediatric evaluation of disability inventory. Ann Ig. 2018;30:153-161. doi:10.7416/ai.2018.2206

71. Berardi A, De Santis R, Tofani M, et al. The wheelchair use confidence scale: Italian translation, adaptation, and validation of the short form. Disabil Rehabil. 2018;13(6):1-6.

72. Tofani M, Candeloro C, Sabbadini M, et al. The psychosocial impact of assistive device scale: Italian validation in a cohort of nonambulant people with neuromotor disorders. Assistive Technol. 2018;1-6. doi:10.1080/10400435.2018.1469553

73. Galeoto G, Berardi A, De Santis R, et al. Validation and cross-cultural adaptation of the Van Lieshout test in an Italian population with cervical spinal cord injury: a psychometric study. Spinal Cord Ser Cases. 2018;4(1):49. doi:10.1038/s41394-018-0083-6

74. Parente M, Tofani M, De Santis R, Esposito G, Santilli V, Galeoto G. The role of the occupational therapist in disaster areas: systematic review. Occup Ther Int. 2017;2017:6474761. doi:10.1155/2017/6474761 